\title{
Effects similar to anxiolysis in an organic extract of Stypopodium zonale on an anxiety related behavior in Drosophila melanogaster
}

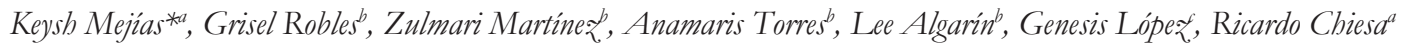 \\ ${ }^{a}$ Department of Biology, University of Puerto Rico, Cayey \\ ${ }^{b}$ General Natural Sciences Program, University of Puerto Rico, Cayey \\ 'Department of Chemistry, University of Puerto Rico, Cayey \\ Students:*keysh.mejias@upr.edu,grisel.robles@upr.edu,zulmari.martinez@upr.edu, anamaris.torres@upr.edu,lee.algarin@upr.edu, \\ genesis.lopez1@upr.edu \\ Mentor: ricardo.chiesa@upr.edu
}

\begin{abstract}
Nature has a great diversity of organisms whose bioactive compounds may potentially be studied. When it comes to aquatic life we find that algae are organisms that are well suited for screening and identification of bioactive compounds due to their widespread distribution in both salt and freshwater. Our hypothesis is that a crude organic extract of the brown algae Stypopodium zonale can decrease anxiety-related behaviors in Drosophila melanogaster. Stypopodium zonale was collected in the south coast of Puerto Rico and the potential anxiolytic-like effects of the extract were studied in an anxiogenic-like behavioral paradigm in Drosophila melanogaster. This behavior is called centrophobia and is measured using an Open Field Arena (OFA). Validation of the paradigm gave the expected results as reported in the literature, in which Drosophila exhibits a phobia (avoidance) of remaining in the center of the OFA, which corresponds to a behavior with anxiety components. The organic extract was dissolved with dimethyl sulfoxide (DMSO). Toxicity tests were performed both for DMSO and the crude organic extract, and neither showed positive results. To perform the behavioral trials, $1 \mathrm{~mL}$ of the crude extract and $4 \mathrm{~mL}$ of water were mixed with $1.8 \mathrm{~g}$ of Drosophila food. The final concentration of the crude extract in the food was $5.4 \mathrm{mg} / \mathrm{mL}$. The adult flies were grown in a tube with the extract until a considerable quantity of larvae was observed, and then the adults were removed. These new larvae, once turned into adult flies, were used for the behavioral trials. The behavior of control flies (food without extract) and experimental flies (extractcontaining food) was recorded with a video camera and the results of the centrophobic behavior were analyzed and compared using quantitative criteria. Both the control and experimental trials were performed in triplicate. The results show that flies grown in food containing the crude extract present a significant reduction in centrophobia compared with control flies. In conclusion, our results suggest that the organic crude extract from Stypopodium zonale has anxiolytic-like effects in a Drosophila melanogaster model with anxiety components. We are currently performing Nuclear Magnetic Resonance (NMR) studies on the crude extracts to identify the most abundant secondary metabolites. Future experiments should include the administration of the crude extracts (or fractions of the most abundant secondary metabolites) to a vertebrate model in to test the effect in a behavior with anxiety components. We are also in the process of developing a preliminary model of possible mechanisms of action of the crude organic extract in the reduction of centrophobia.
\end{abstract}

\section{KEYWORDS}

Anxiety; Algae; Drosophila melanogaster, Open Field Arena; Organic extracts; Centrophobia; Stypopodium zonale

\section{INTRODUCTION}

Algae are aquatic organisms that can be found both in fresh and salt waters. They are classified into classes according to their color, in which three principal groups can be defined: the brown algae (class Phaeophyceae) the green algae (class Chlorophyceae), and the red algae (class Rhodophyceae). In this project, we worked with the Phaeophyceae, specifically the alga Stypopodium zonale, found in the coasts of Puerto Rico. For classification purposes, we followed descriptive and structural criteria described in the book Caribbean Reef Plants. ${ }^{1}$

In the world, there are a total of 1500 species of brown algae; ${ }^{2}$ of these, 65 are found in Puerto Rico. In this project, we used the brown algae Stypopodium zonale, which is easily found in the south coast of Puerto Rico, specifically at Pozuelo Beach in the town of Guayama and Punta Guilarte Beach in the town of Arroyo. 
Algae contains a great variety of bioactive compounds, which is why it is being studied and used for the development of alternative pharmacotherapies to treat different health conditions. ${ }^{3}$ A great diversity of organic compounds with an ample spectrum of biological activity have been isolated from marine algae in the last decades ${ }^{4}$ including compounds with antiinflammatory capacities, antioxidant activity, and antimicrobial activity, among others. ${ }^{5}$ More recently, algal compounds with neuroprotective abilities have also been reported. ${ }^{6}$ Since neuroprotection is an inherent requisite for proper neuronal connectivity in any circuit, including neuronal circuits related to emotions and current drugs for the treatment of anxiety disorders (such as benzodiazepines) have the potential to generate tolerance, dependence, and addiction, it is important to search for alternative chemical agents for the pharmacotherapy of anxiety disorders. Moreover, extracts of the brown seaweed Ecklonia cava evoke anxiolytic-like effect in rats. ${ }^{7}$ These findings set the rationale for this project.

The aim of this project was to assess the potential anxiolytic effects of crude organic extracts obtained from brown algae (class Phaeophyceae) as an alternative pharmacotherapy for the treatment of anxiety disorders. Specifically, we aimed to study the effects of a crude organic extract of the brown algae Stypopodium zonale, the only species of the genus Stypopodium in Puerto Rico. We used the fruit fly Drosophila melanogaster, as the model system for the behavioral studies. Drosophila has been used for decades as a model system for genetics, and more recently it has emerged as a model for the study of neurobiological disorders. ${ }^{8} \mathrm{D}$. melanogaster is neuroanatomically different from humans, but it is also very similar regarding neuronal physiology and neurotransmitters systems. These similarities make Drosophila a versatile and ideal model for the study of behavior and neuropsychiatric disorders. Some examples of the similarities are the neurotransmitter systems, learning behaviors, and memory. ${ }^{9}$ In addition to these similarities in cerebral physiology with the human brain, Drosophila are easily maintained and grown under controlled laboratory conditions.

The paradigm used to generate an anxiety behavior in Drosophila in this project was centrophobia, an innate aversive behavior. ${ }^{10,11}$ Centrophobia is observed with the use of an Open Field Arena (OFA). ${ }^{12}$ The centrophobic behavior can be observed when Drosophila is placed in the center of the OFA and it rapidly moves to the borders, avoiding staying in the middle of the arena. ${ }^{13,14}$ The organic crude extract of Stypopodium zonale was administered in the food from the embryo stage until adulthood, and these adults were used for the behavioral assays.

In summary, the experimental hypothesis is that an organic crude extract of the brown algae Stypopodium zonale will reduce centrophobia in Drosopbila (measured in an OFA). Another important goal of this project is to chemically characterize the secondary metabolites that could be responsible for the anxiolytic effects of the crude extract. A long-term goal of this project is to administer the crude Stypopodium zonale extract and/or purified metabolites to vertebrates, such as rats, in specific brain regions related to anxiety and fear, to assess for any anxiolytic effects.

\section{MATERIALS AND METHODOLOGY}

\section{Collection, Storage, and Classification of Brown Algae}

Two collections of $S$. zonale were made for this project. The first collection of algae was in Pozuelo Beach, in the town of Guayama, Puerto Rico. The second collection was made in Punta Guilarte Beach at Arroyo, Puerto Rico. For both collections, the algae were stored in plastic bags with seawater and were transported to the laboratory surrounded with ice. They were rinsed with filtered sea-water, blotted and air-dried, classified, and stored at $-20^{\circ} \mathrm{C}$.

\section{Extraction of the Organic Compounds}

Two extractions of the algae Stypopodium zonale were made, one in October 2014 and the other in September 2015. To carry out the two extractions, an initial quantity of 150 grams and 35 grams respectively were used to obtain a crude extract yield of 2.20 grams $(2,200 \mathrm{mg})$ and 0.27 grams $(270 \mathrm{mg})$ of organic solids. The organic solids obtained from each extraction were dissolved in a total volume of $10 \mathrm{~mL}$ dimethyl sulfoxide (DMSO) 23\% and 10\%, respectively. The step-by-step process for the organic compound extraction was as follows: algae were crushed in a mortar, triturated in a blender with a mix of dichloromethane and methanol in a 1:1 proportion. After filtering in a vacuum using a flask, a funnel, two filter papers, and celite sand, the filtrate was placed in a distillation flask to evaporate the liquid in a rotary evaporator. The organic solids obtained from the second extraction, dissolved in $10 \mathrm{~mL}$ of DMSO 10\%, were used for the experimental group of the behavioral assays. The concentration of crude extract in the total volume of $10 \mathrm{~mL}$ of DMSO $10 \%$ was $27 \mathrm{mg} / \mathrm{mL}$.

Toxicity Assays with Dimethyl Sulfoxide (DMSO) at 23\%

In order to perform a toxicity assay for DMSO at its highest concentration (23\% DMSO used in the first organic extraction), 16 flies were placed in a tube. This tube contained about $2 \mathrm{~mL}$ of bactoagar $2 \%$ and sucrose $5 \%$, with DMSO at $23 \%$, which solidified at the bottom. The flies were placed under observation for 24 hours. In the first extraction, we were able to dissolve the solids of the crude extracts with a $23 \%$ concentration of DMSO. Although for the second extraction we obtained complete 
dissolution of the solids in the crude extracts using 10\% of DMSO, we tested the toxicity of DMSO at its highest concentration of $23 \%$, for a better assessment of its potential toxic effects.

Preparation of OF A for Assessment of Centrophobia

The Open Field Arena (OFA) was built using two acrylic concentric circles. To cover the circles, a square acrylic top was used. Air flowed into in the circles through four small spaces that remained in each border between the top acrylic cover and the circles. To hold the camera used for the behavioral recordings, a polyvinyl chloride (PVC) tripod was constructed. It consisted of two PVC tubes, one horizontally oriented and the other one vertically oriented. The one placed vertically contained a screw in the superior part to secure the camera, making it stable. Lastly, two $15 \mathrm{Watt}$ flood lights were placed near the OFA, to maintain uniform photo-taxis in Drosophila melanogaster throughout the assay.

\section{Control Assays for the Validation of Centrophobia}

To perform the validation of this paradigm, a triplicate was made, for a total sample size (n) of 35 flies. These flies grew eating regular food, which contains $1.8 \mathrm{~g}$ of Formula 4-24 food (Carolina Biologicals; proprietary mixture) and $5 \mathrm{~mL}$ of water. The flies were immobilized by exposing them to a temperature of $-20^{\circ} \mathrm{C}$ for five minutes. After this, they were moved to the Open Field Arena. As soon as the first fly awoke, their behavior was recorded for 10 minutes without interruption. The criteria used to determine the behavior of centrophobia was the time (in seconds) that the flies remained in the center of the OFA. ${ }^{15}$ As expected, the video recordings showed that once the flies awakened, they immediately began moving towards the borders of the OFA, avoiding staying in the center.

\section{Toxicity Assay with the Organic Extract of Stypopodium zonale}

To assess the potential long-term toxicity of the crude organic extract of Stypopodium zonale, $1.8 \mathrm{~g}$ of Formula 4-24 food were mixed with $4 \mathrm{~mL}$ of water and $1 \mathrm{~mL}$ of the crude extract (at a concentration of $27 \mathrm{mg} / \mathrm{mL}$ ). The final concentration of the crude extracts in the $5 \mathrm{~mL}$ of food was $5.4 \mathrm{mg} / \mathrm{mL}$. The general behavior and sexual behavior of the flies were observed during nine days.

\section{Experimental Centrophobia Assays with the Organic Extract of the Algae Stypopodium ronale}

For the experimental behavioral assays, and to assess the potential anxiolytic effects of the crude extract, a triplicate was made, for a total sample size (n) of 41 flies. The adult flies used had been exposed to $5 \mathrm{~mL}$ of food containing the crude extract at a concentration of $5.4 \mathrm{mg} / \mathrm{mL}$ since embryo stage. The assays were performed as described in Control Assays for the V alidation of Centrophobia. The video recordings showed that flies exposed to the crude extract remained in the center of the OFA for a longer period of time than the control flies. With this, it was concluded that the crude organic extract of the algae Stypopodium zonale has anxiolytic effects on this particular aversive behavior in Drosophila melanogaster.

\section{Chemical Analysis of the Crude Extract from Stypopodium zonale}

After evaporation of the solvent mixture, the flask containing the crude extract from $S$. zonale was placed in a vaccum for one hour to remove any remaining traces of solvents. A spatula was used to obtain a small sample of the extract, place and dissolve it in an NMR vial containing $0.5 \mathrm{~mL}$ of $\mathrm{CDCl}_{3}$ (deuterated chloroform). Once this was done, the vial was transferred to a Bruker Avance NMR spectrophotometer to obtain the 1H-NMR (400 MHz) and 13C-NMR (100 MHz) data.

Statistical analysis of the effects of the crude extract from Stypopodium ronale

Results obtained from both the validation of centrophobic behavior assay and the experimental centrophobic assay with the extract were analyzed using the program Logger Pro 3. The mean and median were obtained for both assays (control and experimental) and their means were compared for statistical significance using a one-tailed student t-test.

\section{RESULTS}

Organic Extractions

Two extractions were made with the Stypopodium zonale algae with a yield of $220 \mathrm{mg} / \mathrm{mL}$ and $27 \mathrm{mg} / \mathrm{mL}$, respectively.

Toxicity Assays with Dimethyl Sulfoxide (DMSO) at 23\%

In the DMSO toxicity assay, no convulsions or deaths were observed. This implies that the DMSO 23\% is not toxic for Drosophila melanogaster.

Toxicity Assay with the Organic Extract of Stypopodium zonale

For the crude organic of $S$. zonale toxicity assay no changes in mating, copulation, oviposition, and development into adulthood were observed. This indicates no long-term toxicity of the crude extract to the Drosophila melanogaster. 
Validation of Anxiety Behavior (Centrophobia)

The anxiety behavior of centrophobia in Drosophila melanogaster when placed in an OFA was successfully validated. The behavior in the OFA of flies exposed to regular food (Table 1A) was registered and compared with the behavior of flies exposed to the crude extract (Table 1B). The mean latency of the flies (time spent in the center of the OFA) was 265 seconds with a standard error of 212 seconds (Figure 2A). The median latency was 236 seconds (Figure 2B).

Behavioral Assays to Assess the Possible Anxiolytic Effects of the Organic Crude Extract of Stypopodium zonale

The flies grown in food containing the crude organic extract of $S$. zonale showed a considerable and statistically significant decrease in centrophobia, remaining for longer times in the center of the OFA. The mean latency of the flies was 467 seconds with a standard error of 180 seconds (Figure 2A). The median latency was 600 seconds (Figure 2B).

\section{Chemical Analysis of the Crude Extract from Stypopodium zonale}

The crude extract of $S$. ronale was analyzed with Nuclear Magnetic Resonance (NMR), which is a powerful technique for the identification of plant metabolites. Analysis of metabolites using NMR is a fast and successful tool to determine the most important regions of the spectrum for further analysis. The NMR analyzed in this study was ${ }^{1} \mathrm{H}-\mathrm{NMR}$ and ${ }^{13} \mathrm{C}-\mathrm{NMR}$. In the ${ }^{1} \mathrm{H}-$ NMR (Figure 1A), signals were present of vinylic protons $(\delta=5.2-5.4 \mathrm{ppm}), \alpha$ - heteroatoms $(\delta=3.0-3.5$ ppm), allylic protons $(\delta=1.5-2.0 \mathrm{ppm})$, and aliphatic protons $(\delta=0.5-1.5 \mathrm{ppm})$. Meanwhile, in the ${ }^{13} \mathrm{C}-\mathrm{NMR}$ (Figure $\left.1 \mathbf{B}\right)$, signals of vinylic carbons $(\delta=130 \mathrm{ppm})$ and aliphatic carbons $(\delta=14-32 \mathrm{ppm})$ were present. These two signals are shown only in the ${ }^{13} \mathrm{C}-\mathrm{NMR}$ spectrum because of the time the extract was analyzed. The ${ }^{1} \mathrm{H}-\mathrm{NMR}$ and the ${ }^{13} \mathrm{C}-\mathrm{NMR}$ spectrums showed signals that are present in cyclic organic compounds. This is common to find in natural compounds, such as terpenoids, according to the scientific literature. ${ }^{16} \mathrm{We}$ are currently working in the purification and characterization of the crude extract.

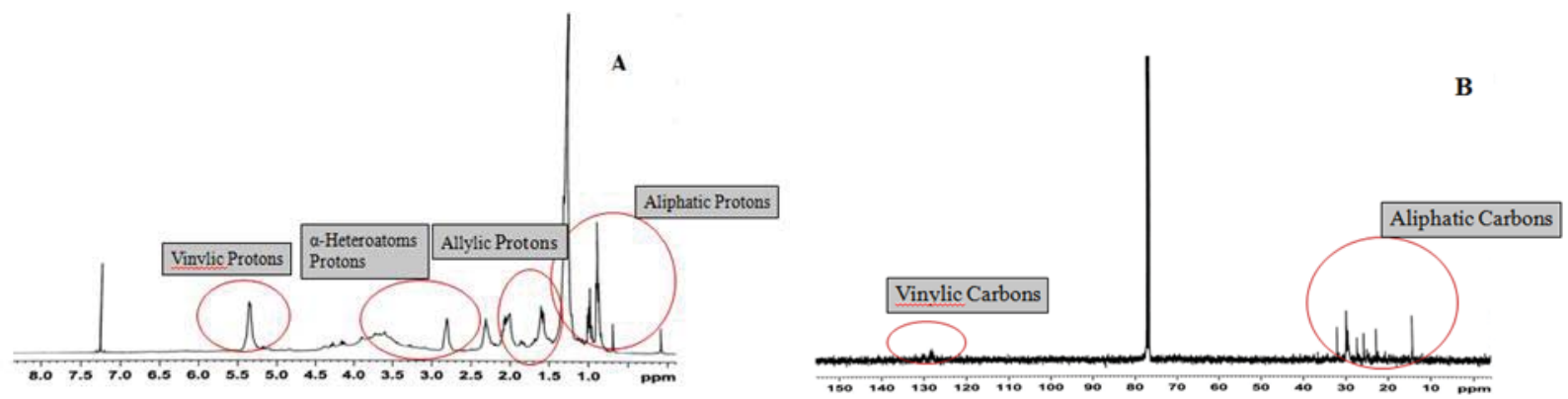

Figure 1. NMR spectra of the crude extract using $\mathrm{CDCl}_{3}$ as a solvent. A. ${ }^{1} \mathrm{H}-\mathrm{NMR}(400 \mathrm{MHz})$ spectrum. B. ${ }^{13} \mathrm{C}-\mathrm{NMR}(100 \mathrm{MHz})$ spectrum.

\section{DISCUSSION}

The objective of toxicity assays was to assess the possibility that the solvent (DMSO) or the $S$. zonale organic extract could affect adult or developing flies. First, a toxicity trial of DMSO $23 \%$ was done, since it was the highest percentage used to dissolve the organic solids. Flies exposed to $23 \%$ DMSO behaved normally and did not convulse or die. Then we assessed the potential toxicity of the crude organic extract of $S$. zonale on a long-term perspective. Adult flies courted and copulated normally, and embryos developed into normal and sexually active adults.

Centrophobia in Drosophila was validated using flies exposed to regular food $(\mathrm{n}=35)$ and then placed in an OFA. The expected avoidance behavior in which the flies move quickly to the walls of the OFA was observed, recorded, and quantified. These flies used for validation of the paradigm also served as the control group. 


\begin{tabular}{|c|c|}
\hline Table 1A & \\
\hline Fly Number & Time (s) \\
\hline 1 & 30 \\
\hline 2 & 34 \\
\hline 3 & 68 \\
\hline 4 & 77 \\
\hline 5 & 77 \\
\hline 6 & 84 \\
\hline 7 & 88 \\
\hline 8 & 88 \\
\hline 9 & 137 \\
\hline 10 & 143 \\
\hline 11 & 160 \\
\hline 12 & 172 \\
\hline 13 & 177 \\
\hline 14 & 188 \\
\hline 15 & 210 \\
\hline 16 & 217 \\
\hline 17 & 217 \\
\hline 18 & 256 \\
\hline 19 & 600 \\
\hline 20 & 600 \\
\hline 21 & 100 \\
\hline 22 & 111 \\
\hline 23 & 128 \\
\hline 24 & 131 \\
\hline 25 & 135 \\
\hline 26 & 160 \\
\hline 27 & 279 \\
\hline 28 & 434 \\
\hline 29 & 589 \\
\hline 30 & 600 \\
\hline 31 & 600 \\
\hline 32 & 600 \\
\hline 33 & 600 \\
\hline 34 & 600 \\
\hline 35 & 600 \\
\hline
\end{tabular}

\begin{tabular}{|c|c|}
\hline \multicolumn{2}{|l|}{ Table 1B } \\
\hline Fly Number & Time (s) \\
\hline 1 & 107 \\
\hline 2 & 116 \\
\hline 3 & 143 \\
\hline 4 & 152 \\
\hline 5 & 172 \\
\hline 6 & 246 \\
\hline 7 & 247 \\
\hline 8 & 249 \\
\hline 9 & 251 \\
\hline 10 & 264 \\
\hline 11 & 271 \\
\hline 12 & 271 \\
\hline 13 & 334 \\
\hline 14 & 450 \\
\hline 15 & 475 \\
\hline 16 & 494 \\
\hline 17 & 558 \\
\hline 18 & 585 \\
\hline 19 & 600 \\
\hline 20 & 600 \\
\hline 21 & 600 \\
\hline 22 & 600 \\
\hline 23 & 600 \\
\hline 24 & 600 \\
\hline 25 & 600 \\
\hline 26 & 600 \\
\hline 27 & 600 \\
\hline 28 & 600 \\
\hline 29 & 600 \\
\hline 30 & 600 \\
\hline 31 & 600 \\
\hline 32 & 600 \\
\hline 33 & 600 \\
\hline 34 & 600 \\
\hline 35 & 600 \\
\hline 36 & 600 \\
\hline 37 & 600 \\
\hline 38 & 600 \\
\hline 39 & 600 \\
\hline 40 & 600 \\
\hline 41 & 600 \\
\hline
\end{tabular}

Table 1. Time spent by the flies in the center of the OFA. A. Flies exposed to regular food and the time each spent (in seconds) in the center of the OFA. Time was measured from the moment the fly moved from the center towards the walls of the OFA. The mean obtained was 265 seconds with a standard deviation of 212 seconds. B. Flies exposed to food with algae extract $(5.4 \mathrm{mg} / \mathrm{mL})$ and the time each spent (in seconds) in the center of the OFA. Time was measured from the moment the fly moved from the center towards the walls of the OFA. The mean obtained was 467 seconds with a standard deviation of 180 seconds. 
Once the centrophobic behavior of control flies was validated, the assays for assessing the potential anxiolytic effects of the crude organic extract of $S$. zonale were performed. The flies used for these assays $(n=41$; Table $1 \mathbf{B})$ were exposed to food containing $5.4 \mathrm{mg} / \mathrm{mL}$ of the crude organic extract since the embryo stage until adulthood. When compared to control flies ( $\mathrm{n}=35$; Table 1A), these treated flies showed a dramatic and statistically significant increase in time spent in the center of the OFA, which translates into a decrease in the centrophobic behavior and indicates an anxiolytic effect of the $S$. zonale organic extract. A quantitative and statistical analysis of the results obtained for both groups (control and treated flies) was performed and is shown in Figure 2A (mean, t-test, p-value) and Figure 2B (median). Based on our toxicity assays, DMSO at the concentrations used in this project is not toxic for the flies. Moreover, there are no reports in the literature of DMSO affecting the behavior of flies on an OFA. In addition, DMSO does not have any effect on the behavior of flies in the dark/light box test or on flies acutely exposed to ethanol. These are two other behavioral and motor activity paradigms we are currently working on in our laboratory.

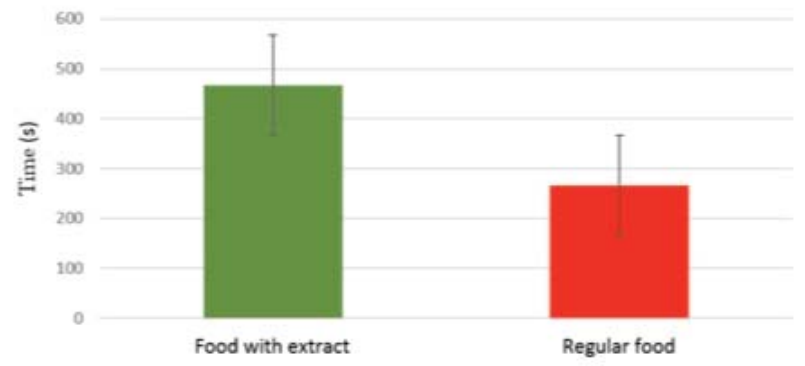

Figure 2A.

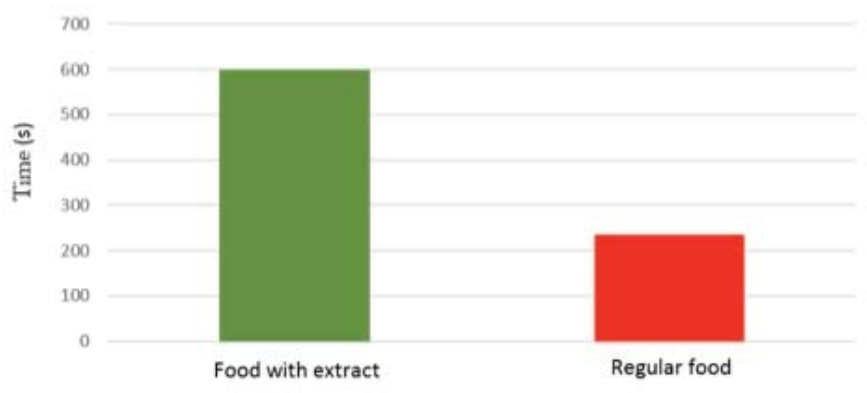

Figure 2B.

Figure 2A. Bar graph of the mean time spent in the center of the OFA by flies exposed to food containing $5.4 \mathrm{mg} / \mathrm{mL}$ of the organic extract of $S$. zonale (green bar; mean $=467$ seconds, S. E. $=180$ seconds) and flies exposed to regular food (red bar; mean $=265$ seconds, S. E. $=212$ seconds), $\mathrm{p}$-value $=0.000013$, which is significant at $\mathrm{p}<0.05$, one-tailed student t-test.

Figure 2B. Bar graphs of the median of the time spent in the center of the OFA by flies exposed to food containing $5.4 \mathrm{mg} / \mathrm{mL}$ of the organic extract of $S$. zonale (green bar; median $=600$ seconds) and flies exposed to regular food (red bar; median $=236$ ).

\section{CONCLUSIONS}

As expected, Drosophila melanogaster grown on regular food (control flies) placed in an OFA show centrophobic behavior. Our results support our experimental hypothesis that flies exposed since the embryo stage to a crude organic extract of the brown algae $S$. zonale show a dramatic and significant decrease in centrophobic behavior. Regarding future projects, protein extracts of $S$. zonale and organic extracts of Sargassum polyceratium (another brown algae) will be tested for their potential anxiolytic effects. In addition, we plan to perform the assays by sex (females only and male only) to asses for differences in behavior and response to the $S$. zonale extract due to variations in the hormonal milieu between sexes. Finally, we aim to perform these experiments, in a vertebrate model, such as rats, to assess the anxiolytic effects of the $S$. zonale crude organic extract or purified metabolites using other anxiogenic and fear-inducing paradigms.

\section{ACKNOWLEDGMENTS}

Our gratitude to Dr. Claudia Ospina, of the Chemistry Department of the UPR-Cayey, for the collaboration in the organic extraction procedure and chemical characterization of the $S$. zonale crude extract. This project was funded by seed money from the FIDI (Institutional Funds for the Development of Research) program, the Dean of Academic Affairs, and from seed money from the Institute of Interdisciplinary Investigations and the BRIC Program of UPR-Cayey. Last, but not least, we give our gratitude to the rest of the research team, whose hard work and dedication made this research project possible. 


\section{REFERENCES}

1. Littler DS and Littler MM. (2000). Caribbean Reef Plants. OffShore Graphics, Inc. Washington, D. C. ISBN 0-9678901-0-1.

2. Raven P, Evert R, Eichhorn S. (1999) Biology of plants. W.H. Freeman and Company/ Worth Publishers. 944.

3. Shalaby, E. (2011) Algae as promising organism for environment and health. Landes Bioscience.

bttp://wmw.ncbi.nlm.nih.gov/pmc/articles/PMC3258063/pdf/psb0609_1338.pdf

4. Matsubara K, Matsuura Y, Hori K, and Miyazawa K. (2000). An anticoagulant proteoglycan from the marine green alga, Codium pugniformis. J. Appl. Phycol. 12: 9-14.

5. Wijesekara I, Yoon N, and Kim S. (2010). Phlorotannins from Ecklonia cava (Phaeophyceae): Biological activities and potential health benefits. BioFactors, 306: 408-414.

6. Pangestuti R, Kim S. (2011) Neuroprotective effects of marine algae. Mar. Drugs, 9: 803-818

7. Suengmok C, Daeseok H, Seon-Bong K, et al. (2012) Depressive Effects on the Central Nervous System and Underlying Mechanism of the Enzymatic Extract and Its Phlorotannin-Rich Fraction from Ecklonia cava Edible Brown Seaweed. Biosci. Biotechnol. Biochem. , 76(1): 163-168

8. Iliadi K. (2009) The genetic basis of emotional behavior: has the time come for a Drosophila model? Journal of Neurogenetics, 23: 136-146.

9. O'Kane C. (2011) Drosophila as a model organism for the study of neuropsychiatric disorders. Pub Med, 7: 37-60.

10. Götz K, Biesinger R. (1985) Centrophobism in Drosophila melanogaster. Journal of Comparative Physiology A, $156(3): 319-327$.

11. Crawley J. (1985). Exploratory behavior models of anxiety in mice. Spring; 9(1):37-44.

12. Roman G, Soibam B, Goldfeder R, Manson-Bishop C, et al. (2012) Modeling Drosophila positional preferences in open field arenas with directional persistence and wall attraction. Plos One, 7(10): e46570

13. Besson M, Martin J. (2005) Centrophobism/thigmotaxis, a new role for the mushroom bodies in Drosophila. Journal of Neurobiology 62: 386-396.

14. Gotz K (1994) Exploratory strategies in Drosophila. Basic Life Sci 16: 391-407.

15. Valente D, Golani I and Mitra P. (2007) Analysis of the Trajectory of Drosophila melanogaster in a Circular Open Field Arena. Plos One 2(10): e1083. DOI: 10.1371/journal.pone.0001083

16. Dorta, E.; Cueto, M.; Brito, I.; Darias, J. (2002). New Terpenoids from the Brown Alga Stypopodium ronale. Nat. Prod. 65, 17271730.

\section{ABOUT THE STUDENT AUTHORS}

Keysh Mejías Martínez is a senior in the Biology Bachelors Program at the University of Puerto Rico at Cayey. She has been working on the article since its beginning and it has been a great experience for the development of her scientific abilities. One of the most satisfying parts of the project was presenting preliminary results at the 56th Annual Drosophila Conference in Chicago in March 2015. Her next goal is to earn an MS Degree in Neurobiology and eventually study medicine.

Grisel Robles Del Valle studies at the University of Puerto Rico at Cayey, and is currently earning a bachelors in the General Natural Sciences program. She has been working on Dr. Ricardo Chiesa's research project for two years. This has helped her to decide on pursuing a Ph.D. degree in a field related to neurobiology. One of her most gratifying experiences was to travel to Chicago for a poster presentation at the $56^{\text {th }}$ Annual Drosophila Conference, in March 2015. She is also is on the board of directors to the student organization called "Clinical Laboratory Professionals", also known as T-MED and is a member of the Microbiology Society of Puerto Rico.

Zulmari Martinez Resto is currently in her last semester of her bachelor's at the University of Puerto Rico at Cayey. She is part of the General Natural Science Program and five other student organizations. She has been part of Dr. Ricardo Chiesa's research team since the beginning of the project and thanks to this great experience, she was able to travel to Chicago for the $56^{\text {th }}$ Annual Drosophila Conference in March 2015. During summer 2014, she participated in Dr. Garcia Rinaldi's Medicine Internship. All of these experiences made her major goal to become a neurosurgeon.

Anamaris Torres Sánchez is currently on her fourth year in the General Natural Science Bachelors Program at the University of Puerto Rico at Cayey. She has been on three different research experiences as an undergraduate. She is also a historian for the $\beta \beta \beta$ National Biological Honor Society Z-E Chapter and is part of three other student organizations such as MEDLIFE. She aspires to pursue graduate studies in nursing and medicine.

Lee Juan Algarín Sepúlveda is a senior in the General Natural Sciences Bachelor's Program at the University of Puerto Rico, in Cayey. In the interest of pursuing higher goals in the fields of research and medicine, he is currently preparing himself by experiencing the life of an undergraduate research student. His biggest desire is to obtain an MD-PhD degree in a neurobiologyrelated field. He is a member of the American Medical Student Association (AMSA) and $\beta \beta \beta$ National Biological Honor Society, Z-E Chapter. 
Genesis López Feliciano is a student at the University of Puerto Rico at Cayey who is doing her bachelor's degree in the Natural Sciences Program with a major in Chemistry. López is working in the Organic Laboratory with her mentor, Claudia Ospina, Ph.D. Currently, López is working in the chemical analysis of the brown algae Stypopodium zonale to isolate new and potentially versatile bioactive compounds. During her bachelor's degree, López has participated in two different research experiences. She is a member of student organizations such as "Círculo de Química"-ACS Chapter, American Society of Biochemistry and Molecular Biology (ASBMB) and Legacy Professional Association.

\section{PRESS SUMMARY}

Nature has a great diversity of organisms with the potential to study their bioactive compounds. When it comes to aquatic life we find that algae are organisms that are understudied, but this does not recognize the capacity for them to be used as potential sources of bioactive compounds with therapeutic capabilities. The hypothesis for this project is that a crude organic extract of the brown algae Stypopodium zonale can decrease anxiety behaviors in Drosophila melanogaster. Stypopodium zonale was collected in the south coast of Puerto Rico. The anxiolytic effects of the extract were studied in an anxiogenic behavioral paradigm in Drosophila melanogaster. To generate an anxiety behavior in Drosophila, we used centrophobia in an Open Field Arena (OFA) as the behavioral paradigm. Validation of the paradigm gave the expected results as reported in the literature, in which Drosophila exhibits a phobia (avoidance) of remaining in the center of the OFA, which corresponds to an anxiety behavior. The organic extract was dissolved with Dimethyl Sulfoxide (DMSO) 10\%. Toxicity trials were performed, and the results obtained eliminated the possibility of any toxicity by the DMSO and the crude extracts. To perform the behavioral trials, the crude extract was placed in $5 \mathrm{ml}$ of water at a concentration of $5.4 \mathrm{mg} / \mathrm{ml}$ as a solution to be mixed afterward with $1.8 \mathrm{~g}$ of Drosophila food. The adult flies were placed in a tube with the extract until a considerable quantity of larvae was observed, and then the adults were removed. These newborn larvae, once turned into adult flies, were used for the behavioral trials. These trials were filmed with a video camera in order to analyze the results of quantitative measurements. After performing the behavioral trials in a triplicate (flies exposed and not exposed to the extract), a comparison of the results was made. Flies grown in food containing the crude extract showed a significant reduction in centrophobia when compared with flies who grew exposed to regular food (control flies). In conclusion, our results suggest that the organic crude extract from Stypopodium zonale has anxiolytic effects in an anxiety model of Drosophila melanogaster. 